\title{
A theoretical model for striped patterns in nematic hydrogels
}

\author{
WU Zhen \& ZHONG Zheng* \\ School of Aerospace Engineering and Applied Mechanics, Tongji University, Shanghai 200092, China
}

Received February 14, 2011; accepted March 23, 2011; published online may 10, 2011

\begin{abstract}
Both nematic elastomers and nematic hydrogels are known to display striped patterns. In contrast to a large body of proposed models for nematic elastomers, few studies exist to interpret striping in nematic hydrogels. In this paper, a theoretical model based on free energy minimization is developed to simulate the drying-induced striping in a layer of nematic hydrogel bonded to a rigid substrate. The liquid crystalline polymers in the nematic hydrogel rotate by certain angles to form the striping in response to a drying environment. Moreover, the striping occurs only for a finite range of humidity, and the boundaries of this range can be tuned.
\end{abstract}

nematic hydrogel, striped pattern, free energy minimization

Citation: $\quad$ Wu Z, Zhong Z. A theoretical model for striped patterns in nematic hydrogels. Chinese Sci Bull, 2011, 56: 1778-1781, doi: 10.1007/s11434-011-4495-y

Liquid-crystalline (LC) polymers can be crosslinked by rubberlike polymers into a three-dimensional polymeric network, known as a LC elastomer [1,2]. Among all possible LC elastomers, nematic elastomers have the simplest symmetry where all the crosslinked LC polymers have a prolate ellipsoidal chain shape with a uniaxial director and are aligned along a common direction. When a nematic elastomer with an affinity for water is in contact with a humid environment, it absorbs a large quantity of water molecules from the environment and swells to a new material known as a nematic hydrogel.

Nematic hydrogels combine the orientational ordering of LC polymers with the hydrophilicity and high-elasticity of hydrogels. This unique combination enables nematic hydrogels to exhibit more novel behaviors than other conventional elastomers. One of these novel behaviors is the formation of striped patterns. This phenomenon was first experimentally observed by Kundler and Finkelmann [3] when they stretched a nematic elastomeric sheet. Subsequently, striped patterns, mainly in nematic elastomers, have been studied by a number of researchers [4-6]. In particular, Fired and Sellers [7] attributed striping to director

*Corresponding author (email: zhongk@tongji.edu.cn) rotations of LC polymers driven by free energy minimization, and developed a simple constitutive model involving two moduli to account for striping in nematic elastomers.

In contrast to a large body of proposed models for nematic elastomers, few analyses exist to interpret striping in nematic hydrogels. This is possibly because of difficulties arising from the coupling of the following three processes: director rotations of LC polymers, large deformations of the polymeric network, and mixing of polymers with water molecules. Coupling of the latter two processes was analyzed by Suo and his collaborators [8-10] in their work on isotropic hydrogels. In this paper, we study striping in a layer of nematic hydrogel bonded to a rigid substrate by proposing a simple form of free energy density that takes the three coupled processes in nematic hydrogels into account.

Figure 1 shows striping in nematic hydrogels. A swollen layer of nematic hydrogel with the director of LC polymers initially aligned perpendicular to the layer is bonded to a rigid substrate (Figure 1(b)). When the external environment is very humid, the hydrogel swells in thickness, enabling the director to remain in the initial perpendicular orientation. As the environment dries, the hydrogel shrinks in thickness, triggering the director to rotate by a certain angle, and thus the striped patterns occur (Figure 1(c)). When the environment is very dry, 
the director rotates to the orientation parallel to the substrate, so that the striped patterns are not observed (Figure 1(d)).

This phenomenon is now transformed into a theoretical model. A stress-free and water-free nematic hydrogel (Figure 1(a)) is taken as the reference state, and the director is assumed to align normal to the layer. Following Fried and Sellers [7,11,12], a symmetric and positive-define conformation tensor $\Lambda$ is used to describe the uniaxial chain shape of the LC polymer in the current state. Likewise, a referential conformation tensor $\Lambda *$ is used to describe the chain shape of the LC polymer in the reference state. The coordinates $X_{1}$ and $X_{2}$ are defined as the material coordinates in the plane of the layer, and $X_{3}$ as the material coordinate normal to the layer. The referential conformation tensor has the form [7,12]:

$$
\boldsymbol{\Lambda}_{*}=\left[\begin{array}{lll}
1 & 0 & 0 \\
0 & 1 & 0 \\
0 & 0 & r
\end{array}\right],
$$

where $r$ is the anisotropic parameter of the chain shape of LC polymer, with $r>1$ being the prolate chain shape, $r<1$ the oblate chain shape, and $r=1$ the isotropic chain shape. The eigenvalues of $\Lambda_{*}$ are assumed to be unaffected by the deformation of the network, so that $\Lambda$ is a rotation of $\Lambda *$. This assumption is approximately valid for temperatures far from the nematic-isotropic transition temperature. When the director rotates by an angle $\theta$ around the $X_{2}$ axis, the conformation tensor in the current state becomes

$$
\Lambda=\left[\begin{array}{ccc}
\cos ^{2} \theta & 0 & \frac{1}{2} \sin 2 \theta \\
0 & 1 & 0 \\
-\frac{1}{2} \sin 2 \theta & 0 & \sin ^{2} \theta+r \cos ^{2} \theta
\end{array}\right] .
$$

It is assumed that, before being bonded to the rigid substrate, the nematic gel swells by an isotropic stretch $\lambda_{0}$ rela-

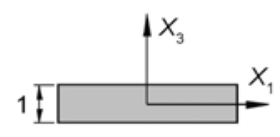

(a) Reference state

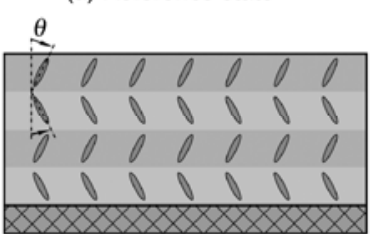

(c) Striped state

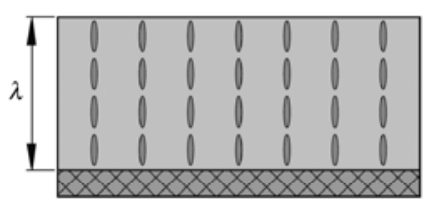

(b) Perpendicular state

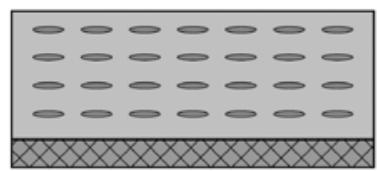

(d) Parallel state
Figure 1 Schematic of striping in a swollen layer of nematic hydrogel bonded to a rigid substrate. (a) In the reference state, the nematic hydrogel is stress-free and water-free. The crosslinked LC polymers (ellipses) have the director orientation normal or parallel to the substrate in the perpendicular state (b) or in the parallel state (d), but rotate by certain angles to form striped patterns in the striped state (c). tive to the reference state. When the nematic hydrogel has been bonded to the substrate, the in-plane stretches are constrained to the initial value $\lambda_{0}$, but the out-of-plane stretch $\lambda$ (i.e. the stretch along the $X_{3}$ axis) can vary with the humidity of the environment (Figure 1(b)). A choice of the deformation gradient that allows for the stretch $\lambda$ and a shear $\delta$ consistent with the director rotations is given by $[7,9]$

$$
\boldsymbol{F}=\left[\begin{array}{ccc}
\lambda_{0} & 0 & \delta \\
0 & \lambda_{0} & 0 \\
0 & 0 & \lambda
\end{array}\right]
$$

Individual polymers and water molecules are taken to be incompressible, so that the change in volume of the hydrogel only arises from absorbing water molecules $[8,10]$ :

$$
v C=\operatorname{det} \boldsymbol{F}-1=\lambda \lambda_{0}^{2}-1,
$$

where $v$ is the volume per water molecule and $C$ is the number of water molecules in the current state per reference volume.

We assume that the free energy $W$ of nematic hydrogels has two contributions as follows:

$$
W=W_{m}+W_{s} .
$$

The contribution from mixing of polymers and water molecules is described by $[10,13]$

$$
W_{m}=-\frac{k T}{v}\left(v C \log \frac{v C+1}{v C}+\frac{\chi}{1+v C}\right),
$$

and the contribution from elastic deformation is described by $[11,12]$

$$
W_{s}=\frac{1}{2} N k T\left(\phi_{1} \operatorname{tr} \boldsymbol{B}+\phi_{2} \operatorname{tr} \boldsymbol{A}-3-2 \log \operatorname{det} \boldsymbol{F}\right),
$$

where $k$ is the Boltzmann constant; $T$ is the absolute temperature; $\chi$ is a dimensionless measure of the enthalpy of mixing; $N$ is the number of polymers per reference volume; $\phi_{1}$ and $\phi_{2}$ relate to the fraction of the number of rubberlike polymers and LC polymers, respectively, in $N$ (so that $\phi_{1}+\phi_{2}=1$ ). The elastic free energy (eq. (7)) involves the following two strain tensors: $\boldsymbol{B}=\boldsymbol{F F}^{\mathrm{T}}$, which is a macroscopic strain tensor accounting for the macroscopic deformation of the polymeric network; and $\boldsymbol{A}=\boldsymbol{\Lambda}^{-1} \boldsymbol{F} \boldsymbol{\Lambda} * \boldsymbol{F}^{\mathrm{T}}$, which is a microscopic strain tensor measuring the deviation of the relative rotation of the director from the macroscopic deformation. A detailed discussion on these two strain tensors is given in [11].

The nematic hydrogel and the environment constitute an isolated thermodynamic system. Since the environment is so large, the chemical potential $\mu$ of the water molecules in the environment is assumed to be constant. When the hydrogel adsorbs a number of water molecules $C$, the free energy of 
the environment decreases by $\mu C$, and accordingly the free energy of the system becomes

$$
G=W-\mu C .
$$

The system has three degrees of freedom, the director rotation $\theta$, the shear $\delta$ and the stretch $\lambda$ in the thickness. Equilibrium states minimize the free energy of the system, which requires that $G$ be stationary with respect to $\theta, \delta$ and $\lambda$, which can be expressed as

$$
\partial G / \partial \theta=0, \quad \partial G / \partial \delta=0, \quad \partial G / \partial \lambda=0 .
$$

Solving eq. (9) for $\theta, \delta$ and $\lambda$, three classes of solutions are obtained that minimize the free energy $G$ as follows:

(1) $\theta=0, \delta=0, \lambda-\frac{1}{\lambda}=\frac{\mu-\hat{\mu}(\lambda)}{k T} \frac{\lambda_{0}^{2}}{N v}$ for $\mu>\mu_{a}$;

(2) $\theta=\frac{\pi}{2}, \quad \delta=0, \quad \lambda\left(\phi_{1}+r \phi_{2}\right)-\frac{1}{\lambda}=\frac{\mu-\hat{\mu}(\lambda)}{k T} \frac{\lambda_{0}^{2}}{N v} \quad$ for $\mu<\mu_{b}$

(3) $\theta_{ \pm}(\lambda)= \pm \sin \frac{\sqrt{\lambda_{a}-\lambda}}{\lambda_{a}-\lambda_{b}}, \delta_{ \pm}(\lambda)= \pm \sqrt{\left(\lambda-\lambda_{a}\right)\left(\lambda-\lambda_{b}\right)}$, $\lambda_{a}-\frac{1}{\lambda}=\frac{\mu-\hat{\mu}(\lambda)}{k T} \frac{\lambda_{0}^{2}}{N v}$ for $\mu_{b} \leqslant \mu \leqslant \mu_{a}$.

Here

$$
\begin{gathered}
\hat{\mu}(\lambda)=k T\left[\log \left(1-\lambda^{-1} \lambda_{0}^{-2}\right)+\lambda^{-1} \lambda_{0}^{-2}+\chi \lambda^{-2} \lambda_{0}^{-4}\right], \\
\mu_{a}=k T N v \lambda_{0}^{-2}\left(\lambda_{a}-\lambda_{a}^{-1}\right)+\hat{\mu}\left(\lambda_{a}\right), \\
\mu_{b}=k T N v \lambda_{0}^{-2}\left(\lambda_{b}-\lambda_{b}^{-1}\right)+\hat{\mu}\left(\lambda_{b}\right),
\end{gathered}
$$

with $\lambda_{a}=\lambda_{0} \sqrt{\left(\phi_{1}+r \phi_{2}\right) / r}$ and $\lambda_{b}=\lambda_{0} / \sqrt{r\left(\phi_{1}+r \phi_{2}\right)}$. The stretch $\lambda$ appearing in each class of solutions is obtained by solving the corresponding nonlinear function with a given value of $\mu$ numerically. The first and the second solutions represent the perpendicular and the parallel states. The third solution accounts for the striped state, where two opposite director orientations $\theta_{ \pm}$coexist with two oppositely oriented shears $\delta_{ \pm}$. The third solution holds only for a limited range between two critical chemical potentials, $\mu_{a}$ and $\mu_{b}$. The chemical potential of water molecules in the environment is related to the relative humidity by $\mu=k T \log R H$ with $k T=4 \times 10^{-21} \mathrm{~J}$ at room temperature. Consequently, the two critical chemical potentials also give two critical humidities, $R H_{a}$ and $R H_{b}$. This indicates that striping occurs only in a finite range of humidity, $R H_{b} \leqslant R H \leqslant R H_{a}$.

Figure 2 plots the director rotation $\theta$ as a function of the humidity of the external environment. In the curve labeled $\lambda_{0}=1.5$ (with $R H_{a}=95 \%$ ), the director remains in the initial perpendicular orientation when the environment is more humid than the critical humidity $R H_{a}$, but rotates by a large angle when the environment dries slightly below $R H_{a}$. Then the director rotation $\theta$ increases gradually in response to a drying environment unless the environment dries to another critical humidity $\mathrm{RH}_{b}$ (approximately $42 \%$ ). When the environment is less humid than $R H_{b}$, the director reaches the parallel orientation and maintains this orientation regardless of any further decrease in the humidity. In the finite range $R H_{b} \leqslant R H \leqslant R H_{a}$ where striping occurs, different levels of humidity give different but specific values of $\theta$.

The critical humidity can be tuned. Figure 3 shows how $R H_{a}$ and $R H_{b}$ vary with the initial stretch $\lambda_{0}$. The range between $R H_{a}$ and $R H_{b}$ decreases substantially as $\lambda_{0}$ increases. When $\lambda_{0}$ is sufficiently large, the gap between the first and the second solutions vanishes, which results in a sharp transition from the perpendicular state to the parallel state. At this limit, striping may not be observed. When $\lambda_{0}$ is relatively small (e.g. $\left.\lambda_{0}<1.4\right), R H_{b}$ reduces to a constant vanishing value. In this case, the parallel state that corresponds

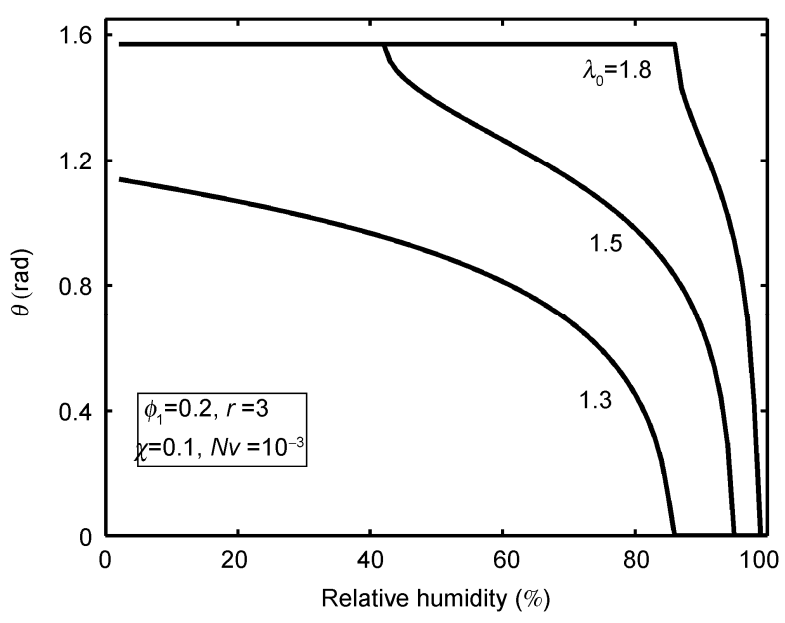

Figure 2 Variation of the director rotation $\theta$ with the relative humidity of the environment at various values of the initial stretch $\lambda_{0}$.

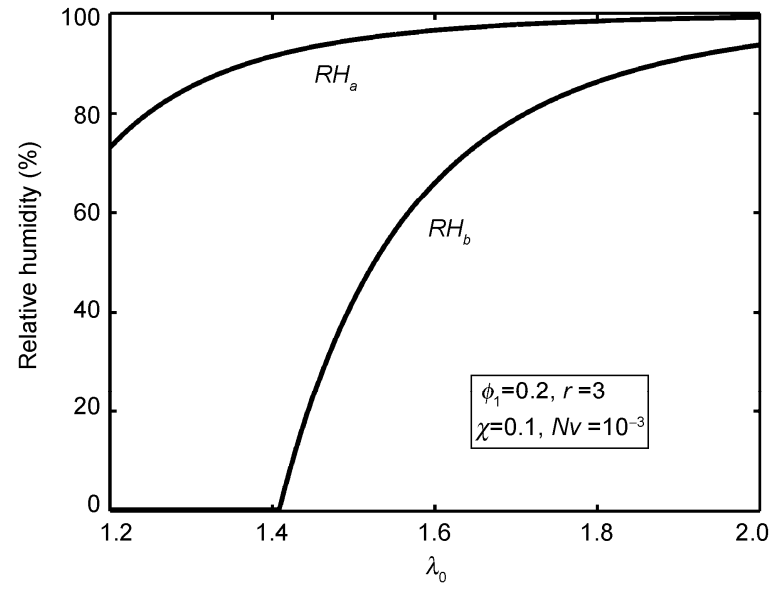

Figure 3 Variation of the critical humidity $R H_{a}$ and $R H_{b}$ with the initial stretch $\lambda_{0}$. 
to the third solution is unreachable, as illustrated by the curve labeled $\lambda_{0}=1.3$ in Figure 2).

In summary, a layer of nematic hydrogel bonded to a rigid substrate allows director rotations associated with striping for a finite range of humidity. Our results show that the bounds of this finite range of humidity can be tuned by choosing nematic hydrogels with different initial stretches. Besides humidity, our model based on free energy minimization can be modified for striping induced by other stimuli [14], which will be an important area of future research. Many types of hydrogels with unique mechanical properties and biocompatibility have been fabricated in recent years [15-17]. In consideration of this, this model should still be applicable when these new properties are incorporated into nematic hydrogels, as long as a specialized free energy function is given.

This work was supported by the National Natural Science Foundation of China (10872150, 11090334) and Shanghai Leading Academic Discipline Project (B302).
1 de Gennes P G. Acad Sci Paris, 1975, 281: 101-103

2 Li M H, Keller P, Yang J Y, et al. Adv Mater, 2004, 16: 1922-1925

3 Kundler I, Finkelmann H. Macromol Rapid Commun, 1995, 16: 679686

4 Verwey G C, Warner M, Terentjev E M. J Phys II France, 1996, 6: 1273-1290

5 Conti S, DeSimone A, Dolzmann G. J Mech Phys Solids, 2002, 50: 1431-1451

6 Verduzco R, Meng G, Kornfield J A, et al. Phys Rev Lett, 2006, 96 : 147802

7 Fried E, Sellers S. J Appl Phys, 2006, 100: 043521

8 Hong W, Liu Z S, Suo Z G. Int J Solids Struct, 2009, 46: 3282-3289

9 Hong W, Zhao X H, Suo Z G. J Appl Phys, 2008, 104: 084905

10 Hong W, Zhao X H, Zhou J X, et al. J Mech Phys Solids, 2008, 56: 1779-1793

11 Fried E, Sellers S. J Mech Phys Solids, 2004, 52: 1671-1689

12 Fried E, Sellers S. J Chem Phys, 2005, 123: 044901

13 Flory P J, Rehner J. J Chem Phys, 1943, 11: 521-526

14 Hesse H C, Beck R, Ding C, et al. Langmuir, 2008, 24: 8397-8401

15 Li L, Lin Z B, Yang X, et al. Chinese Sci Bull, 2009, 54: 1622-1625

16 Zhang D K, Shen Y Q, Ge S R. Sci China Ser E-Tech Sci, 2009, 52: 2474-2480

17 Sun J G, Tang J, Ding J D. Chinese Sci Bull, 2009, 54: 3154-3159

Open Access This article is distributed under the terms of the Creative Commons Attribution License which permits any use, distribution, and reproduction in any medium, provided the original author(s) and source are credited. 